\title{
ON THE EIGENVALUES OF THE LAPLACIAN FOR CERTAIN PERTURBATIONS OF THE STANDARD EUCLIDEAN METRIC ON $S^{2 *}$
}

\author{
ANANDATEERTHA MANGASULI ${ }^{\dagger}$
}

\begin{abstract}
We introduce certain conformal, rotationally symmetric, real analytic perturbations of the standard Euclidean metric on $S^{2}$ and study the perturbed eigenvalues of the Laplace operators for the metrics sufficiently close to the Euclidean metric.
\end{abstract}

Key words. Laplace operator, eigenvalues, Legendre polynomials.

AMS subject classifications. Primary 58J50; Secondary 34B30

1. Introduction. Let $(M, g)$ be a compact Riemannian manifold of dimension $n$. If $\left(U, x^{1}, \ldots, x^{n}\right)$ is a coordinate system on $M,\left(g_{i j}\right)$, the coefficient matrix of the metric $g$ with respect to this coordinate system and $\left(g^{i j}\right)$, the inverse of $\left(g_{i j}\right)$, then the Laplace operator in these coordinates has the expression:

$$
\Delta_{g} f=-\frac{1}{\sqrt{d_{g}}} \sum_{i=1}^{n} \frac{\partial}{\partial x^{i}}\left(\sum_{j=1}^{n} g^{i j} \sqrt{d_{g}} \frac{\partial f}{\partial x^{j}}\right),
$$

for a smooth function $f$ on $M$, where $d_{g}$ is the determinant of the matrix $\left(g_{i j}\right)$. It is well known that the set of eigenvalues of $\Delta_{g}$ consists of a sequence

$$
0=\lambda_{0}<\lambda_{1} \leq \lambda_{2} \leq \lambda_{3} \leq \ldots \ldots \text {. } / \infty
$$

where each eigenvalue is of finite multiplicity and is repeated as many times as its multiplicity in this sequence. The eigenspaces corresponding to distinct eigenvalues are orthogonal to each other in $L^{2}(M)$, and $L^{2}(M)$ is the direct sum of all the eigenspaces (see [2]). A well-known theorem of Lichnerowicz states the following:

THEOREM 1.1 (Lichnerowicz). Let $(N, g)$ be a compact Riemannian manifold of dimension $n$. If there exists a positive number $k$ such that

$$
\operatorname{Ric}_{g} \geq k g,
$$

$\operatorname{Ric}_{g}$ being the Ricci tensor of $g$, then

$$
\lambda_{1}(g) \geq \frac{n}{n-1} k
$$

where $\lambda_{1}(g)$ is the first non-zero eigenvalue of the Laplacian, $\Delta_{g}$ acting on smooth functions on $N$. (see [2])

Let $\lambda_{i}\left(g_{0}\right)$ represent the $i^{\text {th }}$ eigenvalue of the Laplace operator for the standard Euclidean metric, $g_{0}$ on $S^{n}$. All these eigenvalues are explicitly known with $\lambda_{1}\left(g_{0}\right)=n$ (see [2]). Hence, Lichnerowicz's theorem compares $\lambda_{1}(g)$ with $\lambda_{1}\left(g_{0}\right)$ in terms of the constants $k$ and $n$. In particular, if $k=n-1$, it gives $\lambda_{1}(g) \geq \lambda_{1}\left(g_{0}\right)$. A natural

\footnotetext{
* Received August 3, 2007; accepted for publication January 30, 2009.

${ }^{\dagger}$ Indian Institute of Science Education and Research (IISER) Bhopal, ITI (Gas Rahat) Building, Govindpura, Bhopal 462 023, India (anandateertha@gmail.com).
} 
question arises: Does Lichnerowicz's theorem generalize to higher eigenvalues, i.e., is it true that

$$
\lambda_{i}(g) \geq \lambda_{i}\left(g_{0}\right), \text { for all } i \geq 1 ?
$$

In general, it is not true, as shown by an example constructed by Donnelly in dimension four in [3]. Nevertheless, it would still be interesting to know what happens in dimensions two and three, as the kind of construction used in [3] to give a counterexample cannot be adapted to these dimensions. In [6], we have shown that the above inequality is satisfied for left-invariant metrics on $S^{3}$.

In this article, we prove that if the underlying manifold is $S^{2}$ then for a given $j \geq 1$, the above eigenvalue inequality holds for all $i \leq j$ for metrics sufficiently close to $g_{0}$ that are obtained by analytically perturbing $g_{0}$ through rotationally symmetric, conformal metrics. See Theorem 3.11 in Section 3.2.

Another motivation for this study has been to investigate a problem regarding the vector space of harmonic functions with growth conditions on non-compact, complete Riemannian manifolds of dimensions three having non-negative Ricci curvature (see $[3])$.

2. Preliminaries. For $f_{1}, f_{2} \in C^{\infty}\left(S^{2}\right)$, let

$$
\left\langle f_{1}, f_{2}\right\rangle_{0}=\int_{S^{2}} f_{1}(\xi) f_{2}(\xi) d V_{0}(\xi)
$$

where $d V_{0}$ is the volume element of $S^{2}$ with respect to $g_{0} . \Delta_{0}$ is a self-adjoint operator on $C^{\infty}\left(S^{2}\right)$ and its set of eigenvalues is precisely $\{n(n+1) \mid n \geq 0\}$, with $n(n+1)$ of multiplicity $2 n+1$, (see [2]). In the following, we exhibit a complete orthonormal basis of the eigenspace associated with $n(n+1)$, in terms of the coordinates on $S^{2}$ given by

$$
P(t, \theta)=\left(\sqrt{1-t^{2}} \cos \theta, \sqrt{1-t^{2}} \sin \theta, t\right), \text { for }-1<t<1, \text { and } 0<\theta<2 \pi .
$$

If $n=0$, then the eigenfunctions are just the constant functions on $S^{2}$. For a positive integer $j$, let

$$
S_{j, 1}(\theta)=\frac{1}{\sqrt{\pi}} \cos (j \theta) ; S_{j, 2}(\theta)=\frac{1}{\sqrt{\pi}} \sin (j \theta), \text { for } 0 \leq \theta \leq 2 \pi .
$$

Let $P_{n}(t)$ be the polynomial solution of the Legendre's equation

$$
\left(1-t^{2}\right) \frac{d^{2} f}{d t^{2}}-2 t \frac{d f}{d t}+n(n+1) f=0 ;-1 \leq t \leq 1
$$

such that $P_{n}(1)=1$. For integers, $n \geq 0$ and $0 \leq j \leq n$, set

$$
A_{n, j}(t)=c_{n, j}\left(1-t^{2}\right)^{j / 2} P_{n}^{(j)}(t),
$$

where $c_{n, j}=\sqrt{\frac{(n-j) !}{(n+j) !} \frac{2 n+1}{2}}$ and $P_{n}^{(j)}(t)$ is the $j^{t h}$ derivative of $P_{n}(t)$. Then, the set $\left\{\frac{1}{\sqrt{2 \pi}} A_{n, 0}(t), A_{n, j}(t) S_{j, k}(\theta) \mid 1 \leq j \leq n, k=1,2\right\}$ forms an orthonormal basis of the eigenspace associated with $n(n+1)$ with respect to the inner product $\langle,\rangle_{0}$, as shown in $[8]$.

For future reference, we now prove two results regarding Legendre polynomials. 
Lemma 2.1. For $0 \leq j \leq n$,

$$
-\left(1-t^{2}\right) P_{n}^{(j+2)}(t)+2(j+1) t P_{n}^{(j+1)}(t)=(n-j)(n+j+1) P_{n}^{(j)}(t) .
$$

Proof. The proof is by induction on $j$. By the definition of $P_{n}(t)$, we have,

$$
-\left(1-t^{2}\right) \frac{d^{2} P_{n}}{d t}+2 t \frac{d P_{n}}{d t}=n(n+1) P_{n}(t) .
$$

This is the case $j=0$ of the assertion. Assume it is true for some $j \geq 0$; then, we have

$$
-\left(1-t^{2}\right) P_{n}^{(j+2)}(t)+2(j+1) t P_{n}^{(j+1)}(t)=(n-j)(n+j+1) P_{n}^{(j)}(t) .
$$

Differentiating this equation with respect to $t$ and simplifying, we get

$$
\begin{aligned}
-\left(1-t^{2}\right) P_{n}^{(j+1+2)}(t)+2 & (j+1+1) t P_{n}^{(j+1+1)}(t)= \\
& \{n-(j+1)\}\{n+(j+1)+1\} P_{n}^{(j+1)}(t)
\end{aligned}
$$

which proves the assertion for $j+1$.

Lemma 2.2. For $0 \leq j \leq n$,

$$
\int_{0}^{1}\left\{(n-j)(n+j+1)\left(1-t^{2}\right)^{j} P_{n}^{(j)}(t)^{2}-\left(1-t^{2}\right)^{j+1} P_{n}^{(j+1)}(t)^{2}\right\} d t=0 .
$$

Proof. Multiplying equation (2.3) throughout by $\left(1-t^{2}\right)^{j} P_{n}^{(j)}(t)$, we get

$$
P_{n}^{(j)}(t) \frac{d}{d t}\left\{-\left(1-t^{2}\right)^{j+1} P_{n}^{(j+1)}(t)\right\}=(n-j)(n+j+1)\left(1-t^{2}\right)^{j} P_{n}^{(j)}(t)^{2} .
$$

Integrating from -1 to 1 by parts, we get

$$
\int_{-1}^{1}\left(1-t^{2}\right)^{j+1} P_{n}^{(j+1)}(t)^{2} d t=(n-j)(n+j+1) \int_{-1}^{1}\left(1-t^{2}\right)^{j} P_{n}^{(j)}(t)^{2} d t,
$$

which proves the assertion, as the integrand is an even polynomial.

3. Conformal perturbations of the Euclidean metric on $S^{2}$. We note the following important result on the behaviour of the eigenvalues and the eigenfunctions of the Laplacian under a real-analytic perturbation of the metric for a compact Riemannian manifold, (see [1], [4]).

TheOREm 3.1. Let $(M, g)$ be a compact Riemannian manifold and let $g_{s}$ be a family of Riemannian metrics on $M$ depending real-analytically on the parameter $s$, such that $g_{0}=g$. Let $\Delta_{s}$ represent the Laplace operator for the metric $g_{s}$. If $\lambda$ is an eigenvalue of $\Delta_{0}$ of multiplicity $m$, then there exist $m$ scalars and $m$ smooth functions on $M(i=1, \ldots, m): \Lambda_{i}(s)$ and $\Phi_{i}(s)$ respectively, depending real-analytically on the parameter $s$ and such that the following hold:

1. $\Delta_{s} \Phi_{i}(s)=\Lambda_{i}(s) \Phi_{i}(s)$ for all $i$ and $s$.

2. $\Lambda_{i}(0)=\lambda$ for all $i$. 
3. $\left\{\Phi_{i}(s)\right\}$ is an orthonormal set for all $s$ with respect to the inner product $\langle,\rangle_{s}$ given by

$$
\left\langle f_{1}, f_{2}\right\rangle_{s}=\int_{M} f_{1} f_{2} d V_{s},
$$

for $f_{1}, f_{2} \in C^{\infty}(M)$, where $d V_{s}$ is the volume element of $M$ with respect to the metric $g_{s}$.

For a real parameter $s$ in the neighbourhood of 0 , let

$$
g_{s}=\mathrm{e}^{2 \rho_{s}} g_{0}
$$

be a conformal, real-analytic perturbation of the standard Euclidean metric $g_{0}$ on $S^{2}$, so that the family $\rho_{s} \in C^{\infty}\left(S^{2}\right)$ depends real-analytically on $s$ and $\rho_{0}=0$. Let $E_{n}$ denote the eigenspace of $\Delta_{0}$ associated with the eigenvalue $n(n+1)$. Let $m=2 n+1$ and, let $\left\{\phi_{i}\right\}_{1<i<m}$ be any orthonormal basis of $E_{n}$ with respect to the inner product $\langle,\rangle_{0}$. Let $\Lambda_{i}(s)$ and $\Phi_{i}(s)$ be as in Theorem 3.1 for the eigenvalue $\lambda=n(n+1)$. We prove the following theorem:

TheOREM 3.2. With notation as above, let $H$ be the symmetric $m \times m$ matrix whose $(i, j)$-th element is given by

$$
H_{i j}=-2 \lambda\left\langle\dot{\rho}_{0} \phi_{i}, \phi_{j}\right\rangle_{0},
$$

where $\dot{\rho_{0}}$ represents the derivative with respect to $s$ of $\rho_{s}$ at $s=0$. Then, the set of eigenvalues of the matrix $H$ is precisely $\left\{\dot{\Lambda}_{i}(0) \mid 1 \leq i \leq m\right\}$, where $\dot{\Lambda}_{i}(0)$ is the derivative with respect to $s$ of the function $\Lambda_{i}(s)$ at $s=0$.

Proof. We have two orthonormal bases of $E_{n},\left\{\phi_{i}\right\}_{1 \leq i \leq m}$ and $\left\{\Phi_{i}(0)\right\}_{1 \leq i \leq m}$. Let $A=\left(a_{i j}\right)$ be the change of base matrix, so that

$$
\phi_{i}=\sum_{k=1}^{m} a_{k i} \Phi_{k}(0) .
$$

Then the matrices $H$ and $A H A^{t}$ have the same eigenvalues and the $(i, j)$-th element of $A H A^{t}$ is given by

$$
-2 \lambda\left\langle\dot{\rho}_{0} \Phi_{i}(0), \Phi_{j}(0)\right\rangle_{0}
$$

In the following we show that,

$$
A H A^{t}=\operatorname{diag}\left\{\dot{\Lambda}_{1}(0), \dot{\Lambda}_{2}(0), \ldots, \dot{\Lambda}_{m}(0)\right\},
$$

which will prove the required result. We have,

$$
\Delta_{0} \Phi_{i}(s)=\mathrm{e}^{2 \rho_{s}} \Delta_{s} \Phi_{i}(s)=\mathrm{e}^{2 \rho_{s}} \Lambda_{i}(s) \Phi_{i}(s) .
$$

Differentiating with respect to $s$ and evaluating at $s=0$ gives,

$$
\Delta_{0} \dot{\Phi}_{i}(0)=2 \lambda \dot{\rho_{0}} \Phi_{i}(0)+\dot{\Lambda}_{i}(0) \Phi_{i}(0)+\lambda \dot{\Phi}_{i}(0),
$$

Taking the inner product with the eigenfunction $\Phi_{j}(0)$ on both sides of this equation and recalling that $\Delta_{0}$ is a self-adjoint operator, we get

$$
\lambda\left\langle\dot{\Phi}_{i}(0), \Phi_{j}(0)\right\rangle_{0}=2 \lambda\left\langle\dot{\rho_{0}} \Phi_{i}(0), \Phi_{j}(0)\right\rangle_{0}+\dot{\Lambda}_{i}(0) \delta_{i j}+\lambda\left\langle\dot{\Phi}_{i}(0), \Phi_{j}(0)\right\rangle_{0},
$$


where $\delta_{i j}$ is the Kronecker delta. Hence, we get

$$
\begin{gathered}
0=2 \lambda\left\langle\dot{\rho_{0}} \Phi_{i}(0), \Phi_{j}(0)\right\rangle_{0}+\dot{\Lambda}_{i}(0) \delta_{i j} \\
\Rightarrow A H A^{t}=\operatorname{diag}\left\{\dot{\Lambda}_{1}(0), \dot{\Lambda}_{2}(0), \ldots, \dot{\Lambda}_{m}(0)\right\},
\end{gathered}
$$

which implies the assertion of the theorem.

Let $K_{s}$ represent the Gaussian curvature of the metric $g_{s}$. Then from standard theory, as $K_{0}=1$, we have,

$$
K_{s}=\mathrm{e}^{-2 \rho_{s}}+\mathrm{e}^{-2 \rho_{s}} \Delta_{0} \rho_{s} .
$$

Differentiating with respect to $s$ and evaluating at $s=0$, we get

$$
\dot{K}_{0}=\left(\Delta_{0}-2\right) \dot{\rho_{0}},
$$

where " ' represents derivative with respect to $s$.

In the following we restrict our attention to perturbations of the type given in equation (3.1) which are further invariant under rotations about a fixed axis and also satisfy,

$$
\left(\Delta_{0}-2\right) \dot{\rho_{0}} \geq 0, \Delta_{0} \dot{\rho_{0}} \not \equiv 2 \dot{\rho_{0}},
$$

In this article we prove that, for such perturbations, given a positive integer $n, \dot{\Lambda_{i}}(0)>$ 0 , for $1 \leq i \leq 2 n+1$ (see Theorem 3.11). Note from equation (3.2) if, $\left(\Delta_{0}-2\right) \dot{\rho_{0}}$, is moreover strictly positive then, for $s$ positive and sufficiently close to $0, K_{s}>1$.

Choose the orthonormal basis of $E_{n}$ given in Section 2 and denote it by $\left\{\phi_{i} \mid 1 \leq\right.$ $i \leq 2 n+1\}$. We show that the square, symmetric matrix of order $2 \mathrm{n}+1$,

$$
H=-2 n(n+1)\left\langle\dot{\rho_{0}} \phi_{i}, \phi_{j}\right\rangle_{0}
$$

is a positive definite matrix. By Theorem 3.2 this will prove that $\dot{\Lambda}_{i}(0)>0$.

In all the following calculations, we use the parametrization of $S^{2}$ given by equation (2.2) and assume that $\rho_{s}$ is invariant under any rotation of $S^{2}$ about the $z$-axis, for all $s$. From the representation of $\phi_{i}$ 's in these coordinates, it then follows that the matrix $H$ in equation (3.4) is a diagonal matrix. Hence, to show that $H$ is positive definite, it suffices to show that, for $0 \leq j \leq n$,

$$
\int_{-1}^{1} \dot{\rho_{0}}(t)\left(1-t^{2}\right)^{j}\left(P_{n}^{(j)}(t)\right)^{2} d t<0
$$

By hypothesis, $\dot{\rho_{0}}$ being independent of $\theta$ the inequality in relation (3.3) assumes the form

$$
-\left(1-t^{2}\right) \frac{d^{2} \dot{\rho_{0}}}{d t^{2}}+2 t \frac{d \dot{\rho_{0}}}{d t}-2 \dot{\rho_{0}} \geq 0 .
$$

3.1. Some results on the solutions of the non-homogeneous Legendre's equation. The second-order, homogeneous differential equation,

$$
-\left(1-t^{2}\right) \frac{d^{2} y}{d t^{2}}+2 t \frac{d y}{d t}-2 y=0,
$$


has two linearly independent solutions,

$$
y_{1}(t)=t \text { and } y_{2}(t)=-1+\frac{t}{2} \log \left(\frac{1+t}{1-t}\right),
$$

on the open interval $(-1,1)$. As will be clear later, we are basically interested in the solutions of the non-homogeneous differential equation,

$$
-\left(1-t^{2}\right) \frac{d^{2} y}{d t^{2}}+2 t \frac{d y}{d t}-2 y=P(t)
$$

when the right hand side, $P(t)$, is a polynomial. Let $\mathcal{T}$ represent the second-order ordinary differential operator:

$$
\mathcal{T}:-\left(1-t^{2}\right) \frac{d^{2}}{d t^{2}}+2 t \frac{d}{d t}
$$

Then, $\mathcal{T}$ - 2 is a self-adjoint operator on $C^{\infty}([-1,1])$ with respect to the inner product,

$$
\left(\left(f_{1}, f_{2}\right)\right)=\int_{-1}^{1} f_{1}(t) f_{2}(t) d t
$$

For any positive integer $n$, the restriction of the operator $\mathcal{T}-2$ to the finite dimensional vector subspace, $\mathcal{P}_{n}$, consisting of polynomials of degree at most $n$, has kernel, $\operatorname{Ker}\left(\mathcal{T}-\left.2\right|_{\mathcal{P}_{n}}\right)=\{k t \mid k \in R\}$. As a consequence we get the following:

LEMma 3.3. If $P(t)$ is an even polynomial then the differential equation,

$$
-\left(1-t^{2}\right) \frac{d^{2} y}{d t^{2}}+2 t \frac{d y}{d t}-2 y=P(t)
$$

has a polynomial solution.

Lemma 3.4. Let $P(t)$ be an even polynomial and, as guaranteed by Lemma 3.3, let $p(t)$ be any polynomial solution of the differential equation:

$$
-\left(1-t^{2}\right) \frac{d^{2} y}{d t^{2}}+2 t \frac{d y}{d t}-2 y=P(t)
$$

Then,

$$
p(0)=-\int_{0}^{1} t P(t) d t
$$

Proof. The polynomial $p(t)$ satisfies

$$
\frac{d}{d t}\left(-\left(1-t^{2}\right) \frac{d p}{d t}\right)-2 p=P
$$

Multiplying both sides of this equation by $t$, and integrating from 0 to 1 by parts two times, we get

$$
p(0)=-\int_{0}^{1} t P(t) d t
$$


THEOREM 3.5. Let $p(t)$ be the unique solution of the initial value problem:

$$
-\left(1-t^{2}\right) \frac{d^{2} y}{d t^{2}}+2 t \frac{d y}{d t}-2 y=P(t) ; \quad y(0)=-\int_{0}^{1} t P(t) d t ; \quad y^{\prime}(0)=0
$$

where $P(t)$ is an even polynomial. Then, $p(t)$ is an even polynomial and it has the following integral representation:

$$
p(t)=\int_{1}^{t} u P(u) d u-t \int_{0}^{t} P(u) d u-t \int_{0}^{t}\left(\int_{1}^{u} v P(v) d v\right) \frac{d u}{1-u^{2}} .
$$

Proof. Let $q(t)$ be any polynomial solution of the given differential equation. Then, from Lemma 3.4,

$$
q(0)=-\int_{0}^{1} t P(t) d t
$$

Now, let

$$
p(t)=q(t)-q^{\prime}(0) t
$$

Then $p(t)$ is a solution of the given initial value problem and so is the polynomial $p(-t)$. By uniqueness of solutions, we get

$$
p(t)=p(-t)
$$

i.e. $\mathrm{p}(\mathrm{t})$ is an even polynomial. We get the required integral representation of $p(t)$ by employing the method of variation of parameters.

LEMma 3.6. Let $P(t)$ be a non-zero polynomial such that $P(t) \geq 0$ in $[-1,1]$. If $p(t)$ is a polynomial solution of the non-homogeneous, second order differential equation

$$
-\left(1-t^{2}\right) \frac{d^{2} y}{d t^{2}}+2 t \frac{d y}{d t}-2 y=P(t)
$$

then we have the following:

1. If $p(a)=0$, for some $0<a<1$, then $p^{\prime}(a)>0$, where " ' denotes derivative with respect to the variable $t$.

2. If $p^{\prime}(a)=0$, for some $0<a<1$, then $p(a)<0$.

Proof. By hypothesis, we have,

$$
\frac{d}{d t}\left(-\left(1-t^{2}\right) \frac{d p}{d t}\right)-2 p(t)=P(t)
$$

Let $0<a<1$; multiplying this differential equation by $t$, and integrating from $a$ to 1 by parts two times, we get

$$
a p^{\prime}(a)-p(a)=\frac{1}{1-a^{2}} \int_{a}^{1} t P(t) d t .
$$


Thus, if $p(a)=0$, then

$$
p^{\prime}(a)=\frac{1}{a\left(1-a^{2}\right)} \int_{a}^{1} t P(t) d t>0, \text { since } P(t) \geq 0 \text { on }[-1,1],
$$

which proves the first assertion.

On the other hand, if $p^{\prime}(a)=0$, then we get

$$
p(a)=-\frac{1}{1-a^{2}} \int_{a}^{1} t P(t) d t<0,
$$

which proves the second assertion.

TheOREm 3.7. Let $p(t)$ be the unique, polynomial solution of the initial value problem:

$$
-\left(1-t^{2}\right) \frac{d^{2} y}{d t^{2}}+2 t \frac{d y}{d t}-2 y=P(t) ; \quad y(0)=-\int_{0}^{1} t P(t) d t ; \quad y^{\prime}(0)=0,
$$

where $P(t)$ is a non-zero even polynomial such that $P(t) \geq 0$ for $t \in[-1,1]$. Then,

$$
p(t)<0 \text { for all } t \in(-1,1) \Longleftrightarrow p(1) \leq 0 .
$$

Proof. If $p(t)<0$ for all $t \in(-1,1)$, then clearly $p(1) \leq 0$. Conversely, suppose $p(1) \leq 0$. From Lemma 3.4,

$$
p(0)=-\int_{0}^{1} t P(t) d t
$$

Since, by assumption, $P(t)$ is non-negative on $[-1,1]$, this implies

$$
p(0)<0 .
$$

$p(t)$ is an even polynomial; therefore, it suffices to show that $p(t)$ is non-zero on $(0,1)$. On the contrary if $p(a)=0$ for some $a \in(0,1)$, then from the assertions made in Lemma 3.6 we are forced to conclude that $p(1)>0$, a contradiction to our assumption. $\mathrm{Q}$

3.2. Main result. In this section, we prove our main result, Theorem 3.11, using the results obtained in Section 3.1. For $n$, a positive integer and $0 \leq j \leq n$, the polynomial $\left(1-t^{2}\right)^{j}\left(P_{n}^{(j)}(t)\right)^{2}$, where $P_{n}(t)$ is the Legendre polynomial of degree $n$, and $P_{n}^{(j)}(t)$ is its $j^{t h}$ derivative, is non-negative in the closed interval $[-1,1]$ and also an even polynomial. First we prove the following:

Theorem 3.8. For $n$, a positive integer and $0 \leq j \leq n$, let $F_{n, j}(t)$ be the unique solution to the initial value problem:

$$
\begin{gathered}
-\left(1-t^{2}\right) \frac{d^{2} y}{d t^{2}}+2 t \frac{d y}{d t}-2 y=\left(1-t^{2}\right)^{j}\left(P_{n}^{(j)}(t)\right)^{2} ; \\
y(0)=-\int_{0}^{1} t\left(1-t^{2}\right)^{j}\left(P_{n}^{(j)}(t)\right)^{2} d t ; y^{\prime}(0)=0 .
\end{gathered}
$$


Then,

$$
F_{n, j}(t)<0, \text { for } t \in(-1,1)
$$

The proof of this theorem will be obtained as a consequence of the following two rather technical lemmas.

Lemma 3.9. For n, a positive integer,

$$
(n-j)(n+j+1) F_{n, j}(1)=\frac{j}{j+1} F_{n, j+1}(1), \quad 0 \leq j \leq n .
$$

Proof. From the integral representation of $F_{n, j}(t)$, we have,

$$
F_{n, j}(1)=-\int_{0}^{1}\left(1-t^{2}\right)^{j} P_{n}^{(j)}(t)^{2} d t-\int_{0}^{1}\left(\int_{1}^{t} u\left(1-u^{2}\right)^{j} P_{n}^{(j)}(u)^{2} d u\right) \frac{1}{1-t^{2}} d t .
$$

a) For $0 \leq j \leq n$, define

$$
Q_{n, j}(t)=\int_{1}^{t} u\left(1-u^{2}\right)^{j} P_{n}^{(j)}(u)^{2} d u
$$

Then, we have

$$
Q_{n, j}(t)=-\frac{1}{2(j+1)} \int_{1}^{t} \frac{d}{d u}\left\{\left(1-u^{2}\right)^{j+1}\right\} P_{n}^{(j)}(u)^{2} d u .
$$

Integrating by parts on the right hand side, we get

$$
Q_{n, j}(t)=-\frac{\left(1-t^{2}\right)^{j+1} P_{n}^{(j)}(t)^{2}}{2(j+1)}+\frac{1}{j+1} \int_{1}^{t}\left(1-u^{2}\right)^{j+1} P_{n}^{(j+1)}(u) P_{n}^{(j)}(u) d u .
$$

b) From equation (2.3), substituting for $(n-j)(n+j+1) P_{n}^{(j)}(u)$, we have,

$$
\begin{aligned}
& (n-j)(n+j+1) \int_{1}^{t}\left(1-u^{2}\right)^{j+1} P_{n}^{(j+1)}(u) P_{n}^{(j)}(u) d u \\
& =\int_{1}^{t}\left(1-u^{2}\right)^{j+1} P_{n}^{(j+1)}(u)\left\{-\left(1-u^{2}\right) P_{n}^{(j+2)}(u)+2(j+1) u P_{n}^{(j+1)}(u)\right\} d u \\
& =\int_{1}^{t}\left(1-u^{2}\right) P_{n}^{(j+1)}(u)\left\{\frac{d}{d u}\left(-\left(1-u^{2}\right)^{j+1} P_{n}^{(j+1)}(u)\right)\right\} d u .
\end{aligned}
$$

Integrating by parts on the right hand side and using equation (2.3), we get

$$
\begin{aligned}
& (n-j)(n+j+1) \int_{1}^{t}\left(1-u^{2}\right)^{j+1} P_{n}^{(j+1)}(u) P_{n}^{(j)}(u) d u \\
& =-\left(1-t^{2}\right)^{j+2} P_{n}^{(j+1)}(t)^{2}+\int_{1}^{t}\left(1-u^{2}\right)^{j+1} P_{n}^{(j+1)}(u) \frac{d}{d u}\left\{\left(1-u^{2}\right) P_{n}^{(j+1)}(u)\right\} d u \\
& =-\left(1-t^{2}\right)^{j+2} P_{n}^{(j+1)}(t)^{2} \\
& \quad+\int_{1}^{t}\left(1-u^{2}\right)^{j+1} P_{n}^{(j+1)}(u)\left\{2 j u P_{n}^{(j+1)}(u)-(n-j)(n+j+1) P_{n}^{(j)}(u)\right\} d u .
\end{aligned}
$$


Simplifying and recalling the definition of $Q_{n, j+1}(t)$ from equation (3.11), we get

$$
\begin{aligned}
2(n-j)(n+j+1) \int_{1}^{t}\left(1-u^{2}\right)^{j+1} P_{n}^{(j+1)}(u) P_{n}^{(j)}(u) d u= \\
\quad-\left(1-t^{2}\right)^{j+2} P_{n}^{(j+1)}(t)^{2}+2 j Q_{n, j+1}(t) .
\end{aligned}
$$

c) From equation (3.13) and equation (3.12), we get

$$
\begin{aligned}
(n-j)(n+j+1) \frac{Q_{n, j}(t)}{1-t^{2}}= & -\frac{1}{2(j+1)}(n-j)(n+j+1)\left(1-t^{2}\right)^{j} P_{n}^{(j)}(t)^{2}- \\
& \frac{1}{2(j+1)}\left(1-t^{2}\right)^{j+1} P_{n}^{(j+1)}(t)^{2}+\frac{j}{j+1} \frac{Q_{n, j+1}(t)}{1-t^{2}} .
\end{aligned}
$$

Thus,

$$
\begin{array}{r}
-(n-j)(n+j+1)\left\{\left(1-t^{2}\right)^{j} P_{n}^{(j)}(t)^{2}+\frac{Q_{n, j}(t)}{1-t^{2}}\right\} \\
=(n-j)(n+j+1)\left\{-\left(1-t^{2}\right)^{j} P_{n}^{(j)}(t)^{2}+\frac{1}{2(j+1)}\left(1-t^{2}\right)^{j} P_{n}^{(j)}(t)^{2}\right\}+ \\
\frac{1}{2(j+1)}\left(1-t^{2}\right)^{j+1} P_{n}^{(j+1)}(t)^{2}-\frac{j}{j+1} \frac{Q_{n, j+1}(t)}{1-t^{2}} .
\end{array}
$$

Adding and subtracting $\frac{j}{j+1}\left(1-t^{2}\right)^{j+1} P_{n}^{(j+1)}(t)^{2}$ on the right hand side of this equation and simplifying, we get,

$$
\begin{gathered}
-(n-j)(n+j+1)\left\{\left(1-t^{2}\right)^{j} P_{n}^{(j)}(t)^{2}+\frac{Q_{n, j}(t)}{1-t^{2}}\right\} \\
=-\frac{2 j+1}{2 j+2}\left\{(n-j)(n+j+1)\left(1-t^{2}\right)^{j} P_{n}^{(j)}(t)^{2}-\left(1-t^{2}\right)^{j+1} P_{n}^{(j+1)}(t)^{2}\right\} \\
-\frac{j}{j+1}\left\{\left(1-t^{2}\right)^{j+1} P_{n}^{(j+1)}(t)^{2}+\frac{1}{1-t^{2}} Q_{n, j+1}(t)\right\} .
\end{gathered}
$$

Integrating this equation from 0 to 1 and recalling equations (3.10) and (3.11), we get

$$
\begin{gathered}
(n-j)(n+j+1) F_{n, j}(1)= \\
-\frac{2 j+1}{2 j+2} \int_{0}^{1}\left\{(n-j)(n+j+1)\left(1-t^{2}\right)^{j} P_{n}^{(j)}(t)^{2}-\left(1-t^{2}\right)^{j+1} P_{n}^{(j+1)}(t)^{2}\right\} d t+ \\
\frac{j}{j+1} F_{n, j+1}(1) .
\end{gathered}
$$

From Lemma 2.2 the integral on the right hand side is zero, and we get,

$$
(n-j)(n+j+1) F_{n, j}(1)=\frac{j}{j+1} F_{n, j+1}(1),
$$

the required equality.

Lemma 3.10. With notation as above,

$$
F_{n, n}(1)<0 .
$$


Proof. First note that, since $P_{n}(t)$ is a degree $n$ polynomial, its $n^{\text {th }}$ derivative is a non-zero constant, say $k$. From equation (3.10), we have

$$
F_{n, n}(1)=-\int_{0}^{1}\left(1-t^{2}\right)^{n} k^{2}-\int_{0}^{1}\left(\int_{1}^{t} u\left(1-u^{2}\right)^{n} k^{2} d u\right) \frac{1}{1-t^{2}} d t .
$$

Hence,

$$
\frac{1}{k^{2}} F_{n, n}(1)=-\frac{2 n+1}{2 n+2} \int_{0}^{1}\left(1-t^{2}\right)^{n} d t
$$

which proves the claim.

We combine the above two results to arrive at a proof of Theorem 3.8.

Proof of Theorem 3.8. By Theorem 3.7, it suffices to show that, for $0 \leq j \leq n$, $F_{n, j}(1) \leq 0$. Substituting $j=0$ in equation (3.9), we get $F_{n, 0}(1)=0$. From Lemma 3.10 and equation (3.9), we find by induction on $j, F_{n, j}(1)<0$, for $1 \leq j \leq n$.

Finally, we prove the main theorem of this paper.

THEOREM 3.11. Let $g_{s}=\mathrm{e}^{2 \rho_{s}} g_{0}$ be a family of Riemannian metrics on $S^{2}$, such that $\rho_{0}=0, g_{0}$ is the Euclidean metric on $S^{2}$, the family $\rho_{s} \in C^{\infty}\left(S^{2}\right)$ depends real-analytically on the parameter $s$ and each $\rho_{s}$ is invariant under rotations about the $z$-axis. For a fixed non-zero eigenvalue, $\lambda$, of the Laplace operator $\Delta_{0}$ for the standard Euclidean metric $g_{0}$ on $S^{2}$, let the scalars $\Lambda_{i}(s), \dot{\Lambda}_{i}(0)$, and functions $\Phi_{i}(s),(1 \leq i \leq$ $m)$ be as given by Theorem 3.1 for the eigenvalue $\lambda$, where $m$ is its multiplicity. Let $K_{s}$ represent the Gaussian curvature function for the metric $g_{s}$ such that, $\left.\frac{\partial K_{s}}{\partial s}\right|_{s=0} \not \equiv 0$. Then we have,

$$
\left.\frac{\partial K_{s}}{\partial s}\right|_{s=0} \geq 0 \Longrightarrow \dot{\Lambda_{i}}(0)>0, \text { for } 1 \leq i \leq m
$$

Proof. Let $(t, \theta)$ be the coordinates on $S^{2}$ as given in equation (2.2). From the discussion following Theorem 3.2, to prove this result, it suffices to show the following: For any positive integer $n$, and $0 \leq j \leq n$,

$$
\frac{d}{d t}\left(-\left(1-t^{2}\right) \frac{d \dot{\rho_{0}}}{d t}\right)-2 \dot{\rho_{0}} \geq 0 \Longrightarrow \int_{-1}^{1} \dot{\rho}_{0}(t)\left(1-t^{2}\right)^{j} P_{n}^{(j)}(t)^{2} d t<0,
$$

where $\dot{\rho_{0}}$ represents the derivative with respect to $s$ of $\rho_{s}$ at $s=0$, and $P_{n}^{(j)}(t)$, represents the $j^{\text {th }}$ derivative of the Legendre polynomial, $P_{n}(t)$, of degree $n$.

Let $\mathcal{T}$ be the second-order differential operator as defined in equation (3.8). As observed before, $\mathcal{T}-2$ is a self adjoint operator on $C^{\infty}([-1,1])$ with respect to the standard $L^{2}$-inner product, $(()$,$) . Let F_{n, j}(t)$ be as in Theorem 3.8 so that

$$
(\mathcal{T}-2) F_{n, j}(t)=\left(1-t^{2}\right)^{j} P_{n}^{(j)}(t)^{2} .
$$

Then we have,

$$
\begin{aligned}
\int_{-1}^{1} \dot{\rho_{0}}(t)\left(1-t^{2}\right)^{j} P_{n}^{(j)}(t)^{2} d t & =\int_{-1}^{1} \dot{\rho_{0}}(t)\left((\mathcal{T}-2) F_{n, j}(t)\right) d t \\
& =\int_{-1}^{1}\left((\mathcal{T}-2) \dot{\rho_{0}}(t)\right) F_{n, j}(t) d t
\end{aligned}
$$


By assumption, $(\mathcal{T}-2) \dot{\rho_{0}} \geq 0$ with $(\mathcal{T}-2) \dot{\rho_{0}} \not \equiv 0$ and from Theorem 3.8, $F_{n, j}(t)<0$ in $(-1,1)$, for $0 \leq j \leq n$. Hence we get,

$$
\int_{-1}^{1} \dot{\rho_{0}}(t)\left(1-t^{2}\right)^{j} P_{n}^{(j)}(t)^{2} d t<0
$$

the required inequality.

Acknowledgments. The work presented here forms a part of the author's Ph.D. thesis [7], written under the direction of Professor Harold Donnelly at Purdue University. The author wishes to thank him for suggesting the problem and for the several subsequent insightful discussions, which helped in solving the problem. The author would also like to thank the referee for his meticulous reading of the manuscript and for pointing out a fallacy that had sneaked into the Introduction section of the original version.

\section{REFERENCES}

[1] M. Berger, Sur les premières valuers propres des variétés riemanniennes, Compositio Mathematica, 26 (1973), Fasc. 2, pp. 129-149.

[2] M. Berger, P. Gauduchon, and E. Mazet, Le Spectre d'une Variété Riemannienne, Lecture Notes in Mathematics, 194, Springer, Berlin, 1971.

[3] H. Donnelly, Harmonic functions on manifolds of nonnegative Ricci curvature, IMRN, 8 (2001).

[4] T. Kato, Perturbation Theory for Linear Operators, vol. 132, Springer-Verlag N.Y., 1966.

[5] A. Lichnerowicz, Géométrie des groupes de transformations, Dunod (1958).

[6] A. Mangasuli, On the eigenvalues of the Laplacian for left-invariant Riemannian metrics on $S^{3}$, International Journal of Mathematics, 18:8 (2007).

[7] A. Mangasuli, Eigenvalues of the Laplacian for certain Riemannian metrics on $S^{2}$ and $S^{3}$, Ph.D. thesis, Purdue University, USA, 2004.

[8] C. MülLER, Spherical Harmonics, Lecture Notes in Mathematics, 17, Springer-Verlag, 1966. 